Escuela de Ciencias Sociales y Humanidades, UNED, C.R.

URL: http://investiga.uned.ac.cr/revistas/index.php/espiga/index

ISSN: 1409-4002 • e-ISSN: 2215-454X

doi: http://dx.doi.org/10.22458/re.v16i0.1928

\title{
La cara y la cruz de los 40 años de existencia de la Universidad Estatal Distancia
}

\author{
Alonso Rodríguez-Chaves \& Christian Ocampo-Hernández*
}

\author{
Recibido: 16 de agosto, 2017 - Aceptado: 06 de octubre, 2017
}

\section{RESUMEN}

Este ensayo académico de carácter argumentativo invita a dilucidar el proceso de evolución y el trabajo realizado por la Universidad Estatal a Distancia de Costa Rica (UNED) a lo largo de sus cuarenta años de existencia; en particular, el impacto que esta ha tenido en la educación costarricense y la efectividad de la modalidad a distancia en el contexto nacional; principalmente como universidad inclusiva y favorecedora de la expansión de la educación y la movilidad social. En el texto, el lector ahonda en procesos de revisión y mejora continua de la gestión, con la cual la Universidad procura validar su quehacer y vigencia. En el último nivel se deja al descubierto una serie de elementos que obliga y compromete a mejorar y modificar de cara a los nuevos tiempos; de igual modo, se invita a las entidades correspondientes a revisar los recursos económicos que se asignan a esta universidad.

Palabras claves: Costa Rica, UNED, educación a distancia, FEES, tecnología, autoaprendizaje y acreditación.

\section{Formato de citación según APA}

Rodríguez- Chaves, A. y Ocampo-Hernández, C. (2017). La cara y la cruz de los 40 años de existencia de la Universidad Estatal Distancia. Revista Espiga, 16(Número Especial), 52-68. doi: http://dx.doi.org/10.22458/re.v16i0.1928

Formato de citación según Chicago

Rodríguez -Chaves, Alonso y Christian Ocampo- Hernández. «La cara y la cruz de los 40 años de existencia de la Universidad Estatal Distancia». Revista Espiga 16, n. ${ }^{\circ}$ Especial (2017): 52-68. doi: http://dx.doi.org/10.22458/re.v16i0.1928

* M.Sc. Alonso Rodríguez Chaves, Coordinador Cátedra de Historia, Universidad Estatal a Distancia, Costa Rica. Correo electrónico: arodriguez@uned.ac.cr y Msc. Christian Ocampo Hernández, Coordinador Técnicas de Investigación a Distancia, Universidad Estatal a Distancia, Costa Rica. Correo electrónico: cocampo@uned.ac.cr 


\section{A manera de introducción}

La educación constituyó un tema trascendental en el proyecto político de Costa Rica desplegado en la segunda mitad del siglo XX; dentro de esa lógica, brota la propuesta de fortalecer y crear nuevos centros de educación superior, con la intención de dar oportunidad efectiva de estudio a jóvenes de escasos recursos económicos y que vivían en comunidades retiradas.

Pese lo loable que pudo parecer la iniciativa de crear una universidad estatal a distancia en el contexto descrito, en su proceso inicial no resultó fácil y tropezó con un cúmulo de acérrimos enemigos quienes cuestionaron la calidad y certeza del método de y aprendizaje a distancia. No obstante, los defensores de este tipo de educación emprendieron una campaña para convencer y no vencer, pues con esa pretensión intentaron persuadir a la opinión pública sobre los beneficios que aportaría la educación a distancia en sectores vulnerables de la población costarricense.

Luego de un amplio pulso de discusión del proyecto, se creó la Universidad Estatal a Distancia (UNED) en 1977. Sin embargo, el devenir no fue placentero, puesto que el ambiente adverso continuó y las condiciones financieras y administrativas se debatieron entre lo difícil e imposible. Empero, el reordenamiento y valioso esfuerzo institucional ayudó a superar los problemas y consolidar su valioso quehacer.

En el ocaso del siglo XX, la UNED logró cambiar el escepticismo por la esperanza, una dinámica que facilitó la respuesta pertinente y oportuna de aquellos sectores poblacionales históricamente desasistidos por el sistema universitario tradicional. Siguiendo con esa lógica, se fue insertando en espacios casi inexplorados por otras universidades, lo cual catapultó como institución precursora de la movilidad social y benefactora de la población desposeída.

En virtud, la UNED inaugura un nuevo siglo, por formar a miles de profesionales con amplios valores históricos en que se ha fundamentado el Estado Costarricense. Asimismo, por propiciar la enseñanza vinculada al conocimiento universal y la reacción constructiva ante los problemas actuales. Sin ego y arrogancia, ha trascendido por contribuir al compromiso social de estimular la reflexión sobre la realidad e identidad nacional, igual por desempeñar un papel protagónico en el desarrollo de las comunidades; en consecuencia, no es extraño que nadie quede fuera por omisión y mucho menos por falta de acción.

A cuarenta años de existencia, sigue perseverante y con deseos de continuar democratizando la educación superior, por lo que sus aspiraciones no son caducas ni amortizadas. Hoy por hoy, su cultura emprendedora constituye un referente de oportunidades para toda la gente. Por eso... UNED, no es un acto de generosidad, pero si una gran obligación.

\section{El preámbulo de una historia}

La educación se perfiló como un tema prioritario en el proyecto político, económico y sociocultural de Costa Rica; pensado y trazado a partir de la segunda mitad del siglo 
XX. En ese contexto fueron determinantes los cambios acaecidos a nivel constitucional, al igual que la instauración de un nuevo modelo de organización estatal y de desarrollo económico, la explosión demográfica, la aceleración de la urbanización y el surgimiento de la industrialización ${ }^{1}$.

Ante la mayor demanda educativa, se desarrolló y consolidó la democratización de la educación secundaria, expansión de la primaria e introducción de la educación técnica. Se presenta, por tanto, la apertura de centros educativos ubicados en distintos lugares del territorio nacional, situación que permitió ampliar la cobertura a sitios alejados y con ello, asistir a sectores de la población que históricamente habían quedado al margen del sistema educativo costarricense.

A nivel de educación superior, el país no contó con una institución de ese tipo desde el cierre de la Universidad de Santo Tomás (1888); por lo que la creación de la Universidad de Costa Rica en los años cuarenta, vino a solventar una trascendental e imperiosa carestía. Sin embargo, este centro educativo pronto se vio obligado a reformarse (1957) y a enmendar una serie de insolvencias que, para entonces, se hicieron cada vez más evidentes.

Con la llegada de los convulsos años setentas marcados por el debate impulsado por ciertos movimientos sociales, el discurso revolucionario que germinaba en diferentes lugares de América Latina, la explosión demográfica y la reinvención de la sociedad costarricense; devino un periodo de cambios sin precedentes. En general, estos sucesos repercutieron y presionaron de alguna manera a que la educación pública se reformulara y respondiera a las demandas y expectativas que tenía en vilo a la población joven costarricense. Como efecto del proceso, se propuso facilitar el acceso a las personas que, por razones diversas, aún se encontraban excluidas de la formación universitaria, lo cual impulsó la creación de varios centros de educación de ese nivel, pero con lógicas fundacionales diferentes ${ }^{2}$.

Con ese espíritu se fundó el Instituto Tecnológico de Costa Rica (1971) y la Universidad Nacional (1973); las cuales brotaron con la idea de complementar sus quehaceres institucionales entre sí. De este modo, la creación de las nuevas universidades se fundamentó en dos principios básicos: atender a poblaciones relegadas por el sistema educativo superior vigente y brindar soluciones educativas funcionales a las regiones del país. Sin duda, lo que se pretendía era formar profesionales en carreras de corte técnico, tecnológico u otros que hacían falta para satisfacer la oferta laboral o que eran imprescindibles para robustecer el proyecto de desarrollo que había emprendido Costa Rica.

Como parte de las medidas y del proceso de revisión adoptados, se promovieron espacios de reflexión en los cuales, varios sectores de la comunidad nacional discutieron y analizaron con profundidad sobre los problemas y el modelo de educación superior que impulsaban los nuevos tiempos. Inspirado en el compendio de inquietudes y propuestas, se originó el Plan Nacional de Desarrollo de la Educación, el cual replanteó subsanar

1. María, Dengo Obregón, Educación Costarricense (San José: EUNED, 2012), 212-213

2. José, Torres Rodríguez, "Los treinta años del Programa de Estudios Generales en la UNED", Acontecer, mayo-julio del 2008, 25. 
y corregir deficiencias acumuladas por el sistema educativo a través de un conjunto de acciones concretas.

Como efecto de este proceso, durante la administración del Presidente Daniel Oduber Quirós (1974-1978), se impulsó la creación de un nuevo centro de educación superior que brindara formación a distancia y que, en consecuencia, permitiera a jóvenes de comunidades rurales, sitios retirados del área metropolitana y de escasos recursos económicos, realizar estudios universitarios con mayor flexibilidad y autonomía. Se suponía entonces que la incorporación de esta modalidad permitiría a una cantidad numerosa de población proveniente de zonas rurales o con dificultades de proseguir con sus estudios presenciales, acceder a la educación superior; ya que, de esta manera, lo podría hacer desde su casa o desde el lugar que lo considerara pertinente ${ }^{3}$.

En este sentido, debe resaltarse el potencial de la educación a distancia como estrategia organizacional, metodológica y tecnológica. Primero, por facilitar la comunicación entre estudiantes, docentes, contenidos de aprendizaje e institución educativa; aunque todos estos elementos no coincidan en tiempo y lugar. Y segundo, por permitir solventar distancias de tipo geográfico, sociales, económicas y culturales ${ }^{4}$.

Con la ilusión de dar forma a esta "revolucionaria" propuesta educativa en el país, la administración Oduber Quirós gestionó en 1975 a través del Dr. Fernando Volio Jiménez, Ministro de Educación Pública, la ayuda y asesoría de varias entidades y universidades extranjeras. Dentro de ese contexto, sobresale la amplia colaboración que suministraron personeros de la Universidad Nacional de Educación a Distancia (UNED) de España, asimismo, la estrecha relación establecida para esos fines con la Open University (Inglaterra), Fern Universitat (Alemania), Everyman's University (Israel) y la Allama Iqbal Open University (Pakistán); instituciones consideradas referentes internacionales en materia de educación a distancia ${ }^{5}$.

Luego de este arduo proceso de investigación, las autoridades educativas costarricenses quedaron convencidas sobre la efectividad y lo promisorio que sería la modalidad a distancia; por lo que en diciembre de 1975 se dispusieron a nombrar una comisión de alto nivel, a la cual se le asignó la histórica tarea de elaborar la propuesta de creación de la universidad pública a distancia de Costa Rica. El grupo fue conformado por los reconocidos académicos nacionales, Enrique Góngora, Francisco Gutiérrez, Chéster Zelaya, Francisco Antonio Pacheco, Mauro Gutiérrez y Óscar Aguilar Bulgarelli; quienes sabidos de la ingente responsabilidad e importancia de su significado para el país, apuraron en terminar y presentar en corto tiempo, la iniciativa discurrida por el Poder Ejecutivo, conocida como proyecto Ley de Creación de la Universidad Estatal a Distancia, la cual fue finiquitada y entregada a la Asamblea Legislativa para el correspondiente análisis y aprobación el 27 de mayo de 1976 (Asamblea Legislativa, Departamento de Archivo, Decreto n. ${ }^{\circ} 6044$, expediente n. ${ }^{\circ}$ 7581, tomo II, ibídem, ff. 450-451).

3. Daniel Oduber Quirós, "La universidad llegará a todos los jóvenes del país", 10 de marzo de 1977, 2 A.

4. Manuel, Moreno Castalleda, "Educación a distancia, un caleidoscopio para el aprendizaje en la diversidad", en Veinte visiones de la educación a distancia Guadalajara (Guadalajara: UDGVIRTUAL, 2012), 17.

5. Oduber Quirós, "La universidad...". 
En términos generales, el proceso que siguió la iniciativa para la aprobación no fue nada fácil, en tanto estuvo plagado por la animadversión, la desinformación, además de un sinfín de negativas que sectores de la academia y política se encargaron de manipular en el imaginario colectivo costarricense. Evidentemente, recelos y temores mal infundados, en detrimento del proyecto, fortalecieron la idea de que la creación de una universidad de este tipo significaría un retroceso del modelo de enseñanza aprendizaje que se venía construyendo en el plano nacional.

La animosidad se acrecentó cuando se advirtió con insistencia e intención maliciosa, lo nefasto que significaría para la situación financiera del sistema universitario estatal la apertura de otra institución superior. En artículos de opinión de aquel momento, se hizo hincapié en la incertidumbre que esto causaría, ya que se especuló que agravaría la difícil situación financiera de las otras universidades estatales y, en consecuencia, habría una inminente redistribución y atomización de los recursos del Fondo de Financiamiento de la Educación Superior ${ }^{6}$.

Más que argumentos sensatos para justificar la férrea oposición, prevaleció el egoísmo de los detractores, quienes vieron con recelo el potencial de este tipo de universidad en el corto y mediano plazo. A ciencia cierta, un puñado de académicos estimularon encarnizados debates en torno a la seriedad y calidad de la modalidad a distancia; pero detrás de ello existía un sentir oculto en que se miraba como un fuerte competidor y letal amenaza a su estatus quo, así como al de la universidad presencial tradicional en la cual trabajaban y habían cimentado una fructífera carrera académica. Desde esa perspectiva, el diputado Arnoldo Ferreto, el Dr. Claudio Gutiérrez, Rector de la UCR, y la Licda. Hilda Chen Apuy, Presidenta del Consejo Universitario, lideraron y emprendieron una campaña en la que cuestionaron la calidad y efectividad de la educación a distancia o, como algunos solían llamar con eufemismo, "educación por carta" (Asamblea Legislativa, Departamento de Archivo, Decreto n. ${ }^{\circ}$ 6044, expediente n. ${ }^{\circ} 7581$, tomo II, ibídem, ff. 108-110).

Entre tanto, quienes defendían el proyecto reaccionaron con una estrategia diferente y prefirieron dirigir mensajes precavidos en los cuales más que convencer, trataron de informar a la opinión pública sobre los beneficios que traería una universidad a distancia al país. En la misma dirección, se buscó esclarecer toda duda que existiera sobre comentarios los cuales afirmaban que la nueva universidad pretendía competir o sustituir a la educación superior tradicional. Al respecto se afirmó mediante comentarios surgidos en la prensa de la época, el valioso apoyo que sería la apertura de la universidad para la atención de las personas desasistidas por el sistema educativo presencial, así como para la gran cantidad de personas autodidactas que sin ningún acompañamiento pedagógico formal habían adquirido grandes saberes a lo largo de su vida ${ }^{7}$.

Después de un amplio debate y una acalorada discusión de los dictámenes en la Asamblea Legislativa, el voto favorable de 42 diputados emitido el 16 de febrero de 1977 permitió aprobar el tan controversial proyecto de ley, que daba visa de creación de una

6. "La Universidad Estatal a Distancia”, Libertad, 10-16 de junio de 1976, 4-5.

7. Cristián, Rodríguez, "El crimen de la autodidáctica", 2 de julio de 1976, La Nación, 16A. 
universidad "no tradicional" y la primera especializada en enseñanza a distancia en Latinoamérica. Tal y como correspondía, el decreto N.$^{\circ} 6044$ denominado Ley de Creación de la Universidad Estatal a Distancia (UNED) fue finiquitado por el doctor Alfonso Carro el 22 de febrero, y el 3 de marzo, por Daniel Oduber Quirós, Presidente de la República y Fernando Volio Jiménez, Ministro de Educación Pública ${ }^{8}$.

\section{La cruz de los primeros tiempos}

Pese que todo hacía creer que se habían superado todos los escollos habidos y por haber, luego devino uno de los obstáculos y afrentas más difíciles que afectaron el arranque inicial del funcionamiento de la Universidad. Principalmente los de aspectos de índole presupuestario y organizacional, los cuales se convirtieron en los retos más difíciles por vencer; por lo que el mismo Dr. Francisco Antonio Pacheco, quien había asumido funciones como primer Rector de la UNED, la consideró como una situación dramática y "miserable".

Con el fin de acabar con el drama de incertidumbre presentado, las autoridades universitarias recién instaladas tramitaron un pliego de peticiones a las instancias gubernamentales correspondientes para tener un inicio normal de las actividades académicas y administrativas. Entre ellas destaca la solicitud del desembolso de fondos en favor de la UNED; sin embargo, estos no se hicieron efectivos debido a que la recaudación de nuevos impuestos solo beneficiaba a las universidades públicas ya existentes. Otro de los mecanismos pensados para solucionar el problema financiero fue la creación del Timbre de la Educación y la Cultura, el cual suponía que el 20\% de lo recolectado iría para la UNED y el resto para las demás universidades. No obstante, el cobro de este fondo no estuvo debidamente organizado ni concebido por el Banco Central, lo que implicó un serio entrabamiento presupuestario y financiero para todas las universidades durante $1977^{10}$.

Así las cosas, la escasez de recursos económicos se fue agudizando con gran celeridad, situación que amenazó con paralizar el funcionamiento de labores de la Universidad, lo cual sirvió a los enemigos históricos de la institución para sobredimensionar la crisis y, por consiguiente, volver a arremeter con gran morbo e intriga respecto a la pertinencia o no de su existencia ${ }^{11}$.

Empero, todas las posiciones adversas y agoreras en menoscabo de la UNED fueron sorteadas con mucha sabiduría y paciencia, formula que ayudó a salir adelante y evitar lo que a todas luces parecía el inminente caos. Así las cosas, y pese a verse afectada por la asignación de los recursos, como institución no desmayó, por el contario, logró matricular sus primeros estudiantes e iniciar sus labores como universidad cuando se impartieron de manera oficial las primeras tutorías el 1 de julio de $1978^{12}$. Por eso, esta fecha algunos

8. República de Costa Rica, Colección de leyes y decretos (San José: Imprenta Nacional, 1977), 530.

9. Francisco Pacheco Fernández, "Grave crisis financiera obligará a cierre de la UNED", La Nación, 30 de julio de 1977, 2A.

10. “La situación financiera de la UNED”, La Nación, 6 de agosto de 1977, 15A.

11. Pacheco Fernández, "Grave crisis...".

12. "Hoy inicia sus cursos la Universidad a Distancia", La Nación, 1 de julio de 1978, 8A. 
la recuerdan como el día verdadero en que se hace efectivo al público uno de los proyectos históricos más trascendentales de la educación costarricense ${ }^{13}$.

\section{La concreción de una historia}

En las siguientes décadas, la UNED logró vencer los fantasmas del pesimismo y el obcecado escepticismo a través de la consolidación de su quehacer. El discurso elocuente demostró que era capaz de dar respuesta pertinente y oportuna a aquellos sectores de la población para los cuales se había creado, los que históricamente el sistema universitario había eludido. Las anteriores circunstancias revelan por qué durante sus primeros años de existencia la población estudiantil se ubicaba en un rango de edades entre los 28 y los 30 años, así como la obligación que implicó a la institución elaborar programas para tales edades y procedencias.

En particular, el ocaso del siglo XX, fue de incesante apertura y esmero por tener en cuenta a las mujeres en los diferentes proyectos académicos, personas con necesidades educativas, privadas de libertad, adultas mayores, costarricenses en el extranjero, comunidades indígenas, con particularidades culturales o que por dificultades económicas, sociales o geográficas no podían tener acceso al estudio superior ${ }^{14}$. En ese sentido, se fue posicionando como una universidad inclusiva y con sensibilidad social, ya que la presencia e influencia indiscutible en sectores poblacionales abandonados por las demás universidades públicas, le catapultó como institución precursora de la movilidad social y favorecedora de los más desposeídos ${ }^{15}$.

La UNED, con visión clara de su hacer, estableció un diálogo permanente con la comunidad nacional e impulsó a través de procesos multidireccionales la ruptura con la linealidad tradicional. Por consiguiente, se distinguió por apoyar a sectores poblacionales con estudios básicos o sin ellos, a los que les proporcionó capacitaciones y acompañamientos para mitigar las deficiencias cognoscitivas en áreas en las que se perfilaban sumamente potenciales. Con ello ratificó su importancia y afianzó su gestión centrada en proporcionar respuestas concretas en áreas formativas de extensión acordes a necesidades específicas, por ende, a redirigir la oferta académica inicial a temas que ayudarán a solventar las insuficiencias que presentaban las comunidades, como lo constató el Segundo Congreso Universitario, moción $008^{16}$.

Convertida en el referente por antonomasia de la extensión universitaria costarricense, también ejecutó un compendio de acciones con fines sociales, que permitieron la loable misión de alcanzar el añorado sueño de la democratización de la educación ${ }^{17}$. Con

13. Rodrigo Carazo Odio, "Carazo: Consolidar la UNED es tarea de todo el pueblo", La Nación, 2A.

14. "UNED responde a necesidades de comunidades indígenas centroamericanas", Acontecer, mayo-julio de 2008, 23.

15. "Mejoramiento educativo atiende a grupos en estado de indigencia", Acontecer, marzo-mayo de 2008, 12.

16. UNED, Congreso Universitario por un Cambio con Participación Real: Memoria. (San José: EUNED, 2000), 114.

17. “UNED: educación para todos”, Suplemento publicitario especial 'Empresas Exitosas', La Nación, 25 de abril del 2008, 18. 
ese espíritu, se gestó una propuesta, la cual planteó y desarrolló la concesión institucional de becas, prerrogativas que permitieron garantizar la inserción y realización de estudios a las personas que no lo podían hacer por diferentes razones, sino que no obtuvieron matrícula en los demás centros educativos ${ }^{18}$.

\section{El devenir de un nuevo siglo}

La UNED ha marcado la generación de un proceso de enseñanza-aprendizaje en el cual el estudiantado logre el aprendizaje autónomo y significativo como una de sus prioridades trascendentales; a su vez, se aleja del esquema tradicional conformado por estudiante-aula-profesor. Para el alcance del cometido, la cobertura geográfica, la tecnología y la ampliación de la oferta académica han constituido nortes imprescindibles de la ruta de trabajo de las últimas administraciones que ha tenido la $\mathrm{UNED}^{19}$.

En relación con la cobertura geográfica y sus ejes de acción en beneficio de la población en general, se ha ido concretando la apertura de centros universitarios en todo el territorio nacional, lo cual ha ayudado a facilitar el funcionamiento de los servicios que presta el sistema educativo a distancia. Con ese espíritu se han realizado importantes inversiones para la construcción de centros educativos, sedes y demás infraestructura necesaria; además, en su afán de articular esfuerzos, se han instaurado convenios de colaboración con entes locales o regionales para consumar el préstamo o donación de inmuebles para adecuarlos al hacer de la actividad docente ${ }^{20}$.

Esencialmente, esto ha ofrecido una excelente oportunidad para fortalecer la cooperación entre las universidades y diversas entidades, quienes aúnan esfuerzos para aprovechar los recursos dispersos y disponibles; asimismo, para organizarlos y canalizarlos en iniciativas al nivel regional. Se aprecia entonces, el fomento del desarrollo de los centros universitarios como elementos habilitadores para la expansión del conocimiento y la cultura en las regiones del país.

Esto reafirma, en gran medida, el compromiso histórico asumido por la UNED, de tener presencia institucional en las zonas rurales y regiones con mayores dificultades para desarrollarse. Para tales efectos, se abocó a la revisión constante de las experiencias y del trabajo que realizan los centros universitarios, asimismo a su reinvención como unidades claves y estratégicas para la desconcentración del quehacer universitario, la mejora institucional, el desarrollo comunal y la contribución de oportunidades productivas ${ }^{21}$.

Con el posicionamiento de la modalidad a distancia, en las últimas décadas y por las particularidades de la población meta, la institución fue incorporando metodologías y medios más versátiles y flexibles de los que solía usar la educación superior tradicional.

18. "UNED: Universidad de trabajadores", Acontecer, noviembre de 2007-febrero de 2008, 16.

19. UNED, Primer Congreso Universitario. Informes de resultados de los talleres (San José: EUNED, 1988), 9.

20. Juan, Parreaguirre Camacho, "Los centros universitarios en el sistema de educación a distancia" en Congreso internacional: tecnología y educación a distancia, El estudiante de la educación a distancia en la perspectiva de nuevo milenio X, Tomo II” (San José: EUNED, 1999), 482-487.

21. Rodrigo, Arias, Camacho, Informe de labores del Rector 2001 (San José: UNED), 2001, XVII. 
Ello trajo un incremento de la tecnología en el proceso de enseñanza aprendizaje, lo cual permitió aplicar novedosos recursos pedagógicos con los estudiantes ${ }^{22}$.

Lo anterior no es de extrañar, ya que la universidad a lo largo de su existencia ha recurrido al uso de diferentes tecnologías, las cuales en su momento han marcado la pauta y han sido pertinentes para la concreción de la misión institucional ${ }^{23}$. Muestra de este proceso fue el uso inicial del correo tradicional, luego el teléfono, prensa, radio, televisión, videoconferencias y todos los apoyos electrónicos que han aparecido y seguido con celeridad y gran suceso en los últimos tiempos ${ }^{24}$. Sobresale al respecto, el empleo de las plataformas de aprendizaje en línea, especialmente en los últimos años ${ }^{25}$.

Vale indicar, que además de las tutorías impartidas, ha existido una variedad de componentes y posibilidades sonoras, textuales y visuales, que integralmente apoyan el proceso de aprendizaje y han permitido a la universidad cumplir con sus objetivos inspiradores. No obstante, para la UNED siempre ha sido de importancia mantener articulados los distintos medios, métodos y técnicas usadas en la educación a distancia, ya que se convierten en elementos funcionales para la transmisión de conocimientos entre las personas que aspiren a su obtención ${ }^{26}$.

En la actualidad, las Tecnologías de la Información y de la Comunicación (TIC) vienen impactando todos los ámbitos de la vida cotidiana, por lo que su utilización se ha convertido en un hecho común. Así no es de extrañar, la importancia que han adquirido y cómo se han ganado espacios en el contexto educativo de la UNED. El uso de las nuevas tecnológicas ha ayudado a innovar, lo cual permite desarrollar estrategias metodológicas que proporcionan al estudiantado experiencias significativas para el ejercicio profesional futuro.

En este sentido, se ha intensificado el uso de las plataformas de aprendizaje en línea, la radio, los sitios web, las redes sociales, los materiales multimedios, entre otros apoyos que se brindan de manera concatenada y que vinculados logran óptimos resultados en los estudiantes. Haciendo eco de lo apuntado, el uso de nuevas tecnologías como instrumentos de apoyo al proceso enseñanza-aprendizaje de la educación a distancia, ha sido visto principalmente como una alternativa viable para crear, reaccionar, transmitir y democratizar el conocimiento porque desde cualquier lugar se puede trabajar.

En el caso particular, las unidades didácticas se han convertido en un importante recurso que permiten la interacción dialéctica entre la persona y su realidad, No obstante,

22. Edwin, León Villalobos, "Cuadernos del Pensamiento Universitario. Discursos", (Heredia: Departamento de Publicaciones de la Universidad Nacional, 1985), 14.

23. Fernando, Bolaños Baldioceda, "La informática educativa: un reto para la educación a distancia en países en vías de desarrollo", en Congreso internacional: tecnología y educación a distancia VI, Compartiendo experiencias alrededor del mundo (San José: EUNED, Nova South Eastern University, 1995), 124-129.

24. María Ruiz y Claudia Salazar, "Educación virtual: plataforma de aprendizaje y desarrollo regional", en Congreso internacional: tecnología y educación a distancia. El estudiante de la educación a distancia en la perspectiva de nuevo milenio X, Tomo II (San José, EUNED, 1999), 563-570.

25. Francisco, Mora Vicarioli, "Objetos de aprendizaje: importancia de su uso en la educación virtual", Revista Calidad en la Educación Superior, nº 3 (1), 104-118.

26. UNED, “Creación y organización de la Universidad Estatal a Distancia”, San José: CIDREB, 2005, 18. 
estas se han venido revisando con frecuencia, con el fin de producir versiones novedosas y mediadas para facilitar y ajustar el proceso enseñanza aprendizaje. De tal manera se ha afianzado como instrumento vital para las asignaturas y uno de los más importantes de los que se conocen dentro del sistema a distancia de la UNED.

Por lo anterior, la UNED presenta en su accionar unidades didácticas que incluyen diversas actividades académicas que junto a demás apoyos, buscan favorecer la formación reflexiva y la reacción constructiva del estudiantado ante los problemas actuales. En mejor entendido, este y demás materiales disponibles, instan al futuro profesional a obtener un conocimiento universal y a comprender que la especialización no es lo único que razonablemente la Universidad puede ofrecerle.

En general, los materiales y diversos apoyos promueven el autoaprendizaje y desarrollo de habilidades bajo la premisa de "aprender a aprender". De este modo, se estimula en los estudiantes el desarrollo de destrezas tales como comprensión, el análisis, la capacidad de síntesis y argumentación. Dichas competencias son imprescindibles en la formación académica, debido al enorme volumen de información a que están expuestos, y a la rapidez como se generan nuevos saberes.

En relación con la oferta académica, esta se ha ido conviniendo de conformidad con los requerimientos de la sociedad nacional, lo cual ha incidido en que la UNED sea la segunda institución de educación superior estatal con mayor matrícula del país. Así en los últimos años, la cantidad de personas inscritas ha sobrepasado las 30000 en todos sus programas, incluyendo 1000 que suelen participar en las formaciones extendidas por los programas de extensión y cursos libres, los cuales persiguen que las personas sean capaces de desarrollar sus facultades y se integren lo mejor posible a la vida útil del país ${ }^{27}$.

Respecto de esta proyección institucional y su ahínco por mejorar la calidad de vida de diversos sectores de la población, sobresalieron importantes iniciativas de formación y capacitación a líderes comunales, las cuales les ayudarán a ejecutar actividades de concienciación para la protección y conservación sostenida de los valores patrimoniales en todas sus manifestaciones ${ }^{28}$.

La ampliación de la oferta ha sido intensificada a nivel de pregrado, grado, posgrado y extensión universitaria, lo cual ha obligado a permanecer en constante diálogo con diferentes sectores y entidades nacionales. Esa vinculación ha permitido la toma acertada de decisiones, así como facilitar la identificación de las necesidades y áreas del conocimiento que deben atenderse en la oferta. Como parte de ese accionar, se ha concretado varios convenios y entendimientos, entre los que destacan el contraído con el Ministerio de Educación Pública (MEP) y del cual surge el Colegio Nacional de Educación a Distancia (CONED) en el 2005. Dicha instancia mantiene como finalidad la de proporcionar educación secundaria a las personas que no la tienen, de igual modo, funciona como

27. Rodrigo, Arias Camacho. "Informe de labores: gestión 2004", San José: UNED, 2005, 1.

28. UNED, "Lineamientos de Política Institucional 2007-2011”, San José: EUNED, 2007, 6. 
el modelo UNED, en el que el estudiante goza de autonomía para organizarse y administrar los tiempos de estudio según le sea más factible ${ }^{29}$.

En congruencia con la línea histórica de trabajo, los principios fundacionales, reconocida y valorada función social acumulada de coadyuvar a la expansión de la educación y la movilidad social ascendente en el país; la Asamblea Legislativa otorgó a la UNED en el año 2007, la máxima distinción que está en facultad de atribuir, como Institución Benemérita de la Educación y la Cultura de Costa Rica. Sin duda, más que un reconocimiento, esta se interpreta como la ratificación del trabajo desempeñado por la universidad. Así las cosas, la institución mantiene vigente que su trabajo no es un acto de generosidad, sino una obligación de continuar democratizando la educación superior y de seguir siendo un referente de oportunidades para toda aquella persona que la necesite.

\section{La cara de los nuevos tiempos}

Con el fin de ser consecuente con los objetivos reales y fundacionales de la institución y la tradición universitaria pública nacional, cuyos postulados básicos son la democracia, la equidad y el desarrollo; la UNED ha venido desarrollando y profundizando en procesos de revisión y mejora continua de su gestión ${ }^{30}$.

Para ello ha incorporado y consolidado una cultura evaluativa institucional, con la cual pretende validar el quehacer y la vigencia académica; principalmente abocada a examinar los criterios cardinales de la orientación del trabajo, el desempeño de sus funciones y la pertinencia institucional en el contexto actual; lo cual ha derivado ajustes en la entrega de la docencia, reorganización y renovación de funciones de algunas unidades y, quizás lo más importante, la diversificación del conocimiento en favor del estudiantado ${ }^{31}$.

En general, los procesos de autoevaluación y acreditación acaecidos, dejan al descubierto que para subsistir y competir con buen acierto, la Universidad se encuentra obligada y comprometida a progresar en una serie de aspectos en los que instituciones similares a nivel nacional e internacional llevan gran ventaja. De este modo, los caminos por recorrer y perfeccionar de la UNED constituyen un desafío para, principalmente, proyectarse y posicionarse contundentemente en el ámbito académico ${ }^{32}$.

En cuanto a las esferas de acción del quehacer universitario, estas se encuentran muy deshilvanadas, ya que no se logra evidenciar un tejido integral. No obstante, se encuentran respaldadas en un marco de confiabilidad, los efectos y la trascendencia que se

29. Marco, Arce Moya, "La oferta educativa como factor democratizante en una sociedad", en Congreso internacional: tecnología y educación a distancia. El estudiante de la educación a distancia en la perspectiva de nuevo milenio X, Tomo I, (San José: EUNED, 1999), 45.

30. Luis, Vargas Solís, "El papel democratizante de la UNED en el contexto de la crisis social y económica", en Universidad Estatal a Distancia. Segundo Congreso Universitario. Por un cambio con participación real, (San José: EUNED, 2000), 539.

31. Guiselle, Hidalgo Molina y Mayra, Segura Loiza, "Estrategias para el desarrollo de una cultura evolutiva en La UNED de Costa Rica”, Revista Iberoamericana de la Educación Digital, n¹ (2001), http://www. utpl.edu.ec/ried/images/pdfs/vol4-1/estrategias_desarrollo.pdf.

32. Rodrigo, Arias Camacho. "Informe de labores. La UNED en el 2007”, San José: UNED, 2008, 5-7. 
verifican de ellas en las comunidades, invitan a reflexionar sobre la pertinencia de lo que se hace y lo que se debe hacer.

En el ámbito investigativo hay que llevar a cabo procesos de formación que potencien las capacidades de los estudiantes, de acuerdo con el contexto específico de cada uno de ellos y sus comunidades. En razón debe revisarse la incorporación efectiva de la investigación como eje transversal en todo el quehacer universitario. De igual manera resulta importante fomentar un cambio cultural en los centros universitarios, tendiente a la sistematización y usos de la información para la construcción del conocimiento, con el fin de que se desarrollen procesos de investigación acordes con los intereses y problemáticas regionales.

Por otro lado, con la creación de la Vicerrectoría de Investigación se guardaron grandes expectativas e ilusiones; no obstante, pese que las propuestas investigativas desarrolladas hasta el momento son valiosas, se esperarían que las próximas tuvieran una lógica más consistente y cercana con todos los sectores de la academia y la realidad de las comunidades. En caso omiso, se estaría respondiendo al pragmatismo circunstancial y no a la misión histórica de la universidad; tal y cual lo estima Savater, quien se niega a reducir la investigación académica a "estudios incomprensibles alejados de la praxis de la vida diaria".

En relación con la extensión social, este ha sido el nicho y quehacer académico histórico que ha distinguido por antonomasia a la UNED, de ahí que nunca puede desmayar en la filosofía institucional y en la de sus funcionarios, el espíritu de servicio y sensibilidad social. Por consiguiente, debe prevalecer la contribución al desarrollo cultural y técnico que se realiza mediante programas y proyectos de extensión a la sociedad costarricense ${ }^{33}$.

Entre otros retos ingentes aparece la diversificación y actualización de la oferta académica, la cual prometa alternativas más actuales y atractivas; pero más que eso, posibilidades efectivas para superar la pobreza que se ha incrementado en el país. En ese afán, no hay que olvidar que la sociedad requiere profesionales formados con principios humanísticos y preocupados por mejorar la calidad de vida del entorno local y nacional ${ }^{34}$.

Lo anterior invita a repensar, que por la naturaleza y razón de ser de la Universidad, todo quehacer, sin excepción alguna, debe estar vinculado y contextualizado a las dinámicas comunales. Por consiguiente, hay que profundizar más el concepto de academia pública al nivel institucional, para comprender que está para ayudar a resolver con sentido crítico los problemas y necesidades locales y nacionales.

A lo largo de su existencia, la Universidad ha transitado e incorporado tecnologías pertinentes y oportunas para generar cambios sustantivos en el proceso de enseñanzaaprendizaje. Este trabajo de más de cuatro décadas ha modificado el paradigma de la educación universitaria costarricense, al procurar desarrollar las capacidades endógenas del estudiante ${ }^{35}$. A través de la incorporación progresiva del aprendizaje en línea, los

33. "La UNED: un nuevo reto académico", Acontecer Universitario, mayo-junio de 1992, n¹8, p. 9.

34. Torres Rodríguez, "Los treinta...".

35. Bolaños Baldioceda, "La informática...", 124-129. 
estudiantes han logrado un aprendizaje autónomo, con el cual han adquirido conocimientos sobre temas sociales, económicos, políticos, culturales, entre otros ${ }^{36}$.

Para algunos, la UNED contribuye, mediante la virtualidad, a la democratización y universalización de la educación y del conocimiento; pues supone que basta con estos medios tecnológicos para dar posibilidad y accesibilidad efectiva a la educación a personas que así lo requieran. No obstante, es importante tomar en consideración la implementación de programas computacionales y apoyos didácticos innovadores y de mejor calidad, pues muchas veces no son bien apreciados y evaluados por los estudiantes, ya que no están incidiendo de manera decidida, en el mejoramiento de su rendimiento.

Contar con el espíritu de liderazgo y compromiso de los profesores para lograr aprendizajes significativos de los estudiantes, no basta. Sin duda, la calidad de los profesores con posiciones muy diversas debe tomarse como una garantía de la generación de conocimientos; sin embargo, estos funcionarios carecen de apoyo y de incentivos para mejorar su nivel académico o actualización profesional. No obstante, no hay espacios para el diálogo académico en el cual puedan intervenir e intercambiar la sistematización de las experiencias, curiosidades y planteamiento de posiciones personales que le ayuden a mejorar el quehacer académico y a tener mejores competencias para formar a futuros profesionales.

Muy de la mano con lo expuesto, una de las mayores tareas por resolver como institución de educación superior pública, es la extrema supeditación en la gestión de lo administrativo sobre la academia. Situación que, en los últimos años, se ha entronizado mediante una corriente tecnócrata en que especialistas con conocimientos y experiencia en el marco de la gestión pública o afines, dan extrema preponderancia a los aspectos presupuestarios y administrativistas, dejando en segundo plano las convicciones históricas de la Universidad y las mejoras significativas a desarrollar en el sistema de enseñar y aprender. Este pragmatismo referido, trata de una perorata, en la cual, el éxito es signado y solo demostrable mediante resultados cuantificables y mediciones racionales. En ese sentido, se han establecido medidas o políticas de corte empresarial donde miran a los estudiantes como clientes, lo cual desnaturaliza la esencia de la UNED.

Particularmente, la mayor preocupación de esta supeditación radica, en que a la academia se le suele excluir, de manera implícita, a la hora de decidir sobre algunas propuestas o proyectos sustanciales que le atañen o afectan a la lógica intrínseca de una universidad de carácter pública. Con poco protagonismo decisorio, urge un cambio en la cultura organizacional y en la gestión institucional, que revierta la situación y en la que el aparato administrativo se limite en acompañar los procesos liderados por el sector académico.

De cara a los nuevos tiempos, la Universidad ha podido consolidar robustas herramientas para lograr superar cualquier escollo que se presente; sin embargo, la escasez o dislocación de los recursos que se mira en la gestión podría convertirse en un vil obstáculo, el cual amenace la generación de estrategias y programaciones académicas que se quieran emprender. Por consiguiente, esto puede ser nefasto, ya que podría socavar la promoción institucional, el desarrollo educativo y la calidad de atención de los estudiantes ${ }^{37}$.

36. Ruiz y Salazar, "Educación virtual...", 563-570.

37. "Aprobada creación de Vicerrectoría de Investigación", Acontecer, marzo-mayo del 2008, 5. 
A manera de balance, el colosal hacer de la UNED con tan pocos recursos es digna de admirar; pero también urge, por justicia y los resultados fehacientes demostrados, una revisión de presupuesto para que siga vigente y prometedora como siempre. En razón, es necesario revisar el presupuesto que emana el Fondo Especial para la Educación Superior (FEES), en tanto resulta paradójico que sea una de las universidades que más estudiantes tienen, con mayor cobertura y que sea la que menos recursos financieros obtiene. Sin duda, preocupa la estabilidad financiera, y en consecuencia, la capacidad para seguir afrontando el crecimiento que ha tenido en los últimos años. En este caso si no hay más dinero en las arcas del Estado, urge enmendar la distribución desigual del Fondo y de los recursos entre las universidades, de conformidad a los resultados obtenidos, a la marcha de sus quehaceres institucionales y al protagonismo demostrado en el escenario nacional e internacional ${ }^{38}$.

En virtud de lo anterior, es titánico como la Universidad Estatal a Distancia (UNED), pese a su encogida y agobiante situación presupuestaria, sigue destacando en todos sus quehaceres. Más aún es encomiable su actitud y gran disposición de seguir colaborando sin escapismos, en las diferentes acciones e iniciativas que se emprenden para mejorar de manera significativa, programas y proyectos relacionados con la formación de decenas de miles de personas ${ }^{39}$.

\section{A manera de conclusión}

La UNED en su trabajo de más de cuatro décadas ha modificado el paradigma de la educación universitaria costarricense, al procurar desarrollar las capacidades endógenas de sus estudiantes. Si bien, ha logrado convencer con creces, sobre la efectividad y promisorio que es la modalidad a distancia, el camino que ha seguido no ha sido nada fácil, en tanto ha tenido que esquivar la animadversión de acérrimos enemigos entre un sinfín de adversidades que fueron apareciendo.

Empero, todas las situaciones adversas en menoscabo de su trabajo han sido sorteadas con ingente acierto, y por el contario, le han ayudado a fortalecerse como institución y todo su accionar, convirtiéndola en uno de los proyectos históricos más trascendentales de la educación costarricense.

En general, en sus primeras décadas, la UNED logró vencer los fantasmas del pesimismo y el obcecado escepticismo a través de la consolidación de su quehacer; asimismo, a través del discurso elocuente y la dinámica tan particular, demostró que era capaz de dar respuesta pertinente y oportuna a aquellos sectores de la población para los cuales había nacido y que históricamente el sistema universitario había marginado.

En el ocaso del siglo XX, se terminó de posicionar como una universidad inclusiva y con sensibilidad social; ya que su presencia indiscutible en el panorama nacional, le catapultó como institución precursora de la movilidad social y favorecedora de los más desposeídos.

38. Esteban, Arrieta Arias, "Nuestra misión es democratizar el conocimiento", La Prensa Libre, 14 de setiembre de 2005, 10 .

39. Rodrigo, Arias Camacho. "Informe de labores. La UNED en el 2007”, San José: UNED, 2008, vi-vii. 
Convertida en el referente por antonomasia de la extensión universitaria costarricense, también destaca por ejecutar un compendio de acciones con fines sociales. Aunado a eso, la UNED amplió su cobertura geográfica, la intensificación de la tecnología y la ampliación de la oferta académica, lo que le permitió sellar el añorado sueño de la democratización de la educación.

En congruencia con la línea histórica de trabajo, los principios fundacionales, reconocida y valorada función social acumulada de coadyuvar a la expansión de la educación y la movilidad social ascendente; no fue extraño que la Asamblea Legislativa le otorgara en el año 2007, la máxima distinción que el Estado Costarricense atribuye, Institución Benemérita de la Educación y la Cultura.

Las cosas no quedaron ahí y con el fin de ser consecuente con los objetivos reales de la institución y los principios de una universidad pública nacional, la UNED ha continuado profundizando procesos arduos de revisión y mejora continua de su gestión. Para lo cual ha consolidado una cultura evaluativa institucional, con la cual pueda validar el quehacer y su vigencia académica.

No obstante, estos procesos de autoevaluación y acreditación acaecidos, dejan al descubierto que para subsistir y seguir compitiendo con acierto, se encuentra obligada y comprometida a progresar en una serie de aspectos en los que instituciones similares a nivel nacional e internacional llevan gran ventaja. De este modo, los caminos por recorrer constituyen un desafío, puesto que son muchas y variadas las tareas por resolver como institución de educación superior pública.

De cara a los nuevos tiempos, la Universidad se encuentra dispuesta a superar cualquier escollo como siempre lo ha hecho, sin embargo, la escasez o dislocación de los recursos se mira como uno de los mayores obstáculos por superar. No obstante, urge por sus resultados fehacientes la revisión y reasignación presupuestaria, para que siga vigente y tan prometedora.

\title{
ABSTRACT
}

\section{The Heads or Tale of the 40-year Existence of Distance State University of Costa Rica}

\begin{abstract}
This academic and argumentative paper invites to explain the process of evolution and the work carried out by Distance State University of Costa Rica through their forty years of existence. Particularly in the impact it has had in the Costa Rican education and the effectiveness of the distance education method in the national context; mainly as an inclusive institution in favor of expansion of education and social mobility. In the text, the reader will deepen in the processes of revision and constant improvement of the management, which validates its accomplishments and goals. In the last level of the article, a series of elements are uncovered. Those elements force and compromise the Institution to improve and modify so that it faces the new era. Also, the pertinent organizations are invited to review the economic resources assigned to the University in the same way.
\end{abstract}

Key words: Costa Rica, UNED, distance education, FEES, technology, self-learning, accreditation. accreditation. 


\section{RÉSUMÉ}

\section{Le côté pile et le côté face des $\mathbf{4 0}$ ans de l'existence de l’Universidad Estatal a Distancia}

Cet essai académique à caractère argumentatif invite à élucider le processus de l'évolution de l'Universidad Estatal a Distancia (UNED) -Université de l'État à Distance - et le travail réalisé tout au long de ses quarante ans d'existence, notamment son impact dans l'éducation costaricienne et l'efficacité de la modalité d'enseignement à distance dans le contexte national. Principalement comme université inclusive qui favorise la démocratisation de l'éducation et la mobilité sociale. À travers le texte, le lecteur approfondit le processus de révision et l'amélioration continue de la gestion avec lequel l'Université s'efforce de valider son travail et son vigueur. Pour terminer, il est mis en lumière une série d'éléments qui oblige et engage à l'amélioration et modification d'après l'air du temps ; de même les entités concernées sont invitées à revoir les ressources économiques allouées à cet université.

Mots clés: Costa Rica, UNED, enseignement à distance, FEES, technologie, autoapprentissage et accréditation.

\section{Bibliografía}

«Aprobada creación de Vicerrectoría de Investigación». Acontecer, marzo-mayo del 2008.

Arce Moya, Marco. «La oferta educativa como factor democratizante en una sociedad», en Congreso internacional: tecnología y educación a distancia. El estudiante de la educación a distancia en la perspectiva de nuevo milenio X, Tomo I. San José: EUNED, 1999.

Arias Camacho, Rodrigo. Informe de labores del Rector 2001. San José: UNED, 2001.

Arias Camacho, Rodrigo. Informe de labores: gestión 2004. San José: UNED, 2005.

Arias Camacho, Rodrigo. Informe de labores. La UNED en el 2007. San José: UNED, 2008.

Arrieta Arias, Esteban. «Nuestra misión es democratizar el conocimiento», La Prensa Libre, 14 de setiembre de 2005.

Bolaños Baldioceda, Fernando. La informática educativa: un reto para la educación a distancia en países en vías de desarrollo, en Congreso internacional: tecnología y educación a distancia VI, Compartiendo experiencias alrededor del mundo. San José: EUNED, Nova South Eastern University, 1995.

Carazo Odio, Rodrigo. «Carazo: Consolidar la UNED es tarea de todo el pueblo». La Nación.

Dengo Obregón, María. Educación costarricense. San José: EUNED, 2012.

Hidalgo Molina, Guiselle y Segura Loiza, Mayra, «Estrategias para el desarrollo de una cultura evolutiva en La UNED de Costa Rica». Revista Iberoamericana de la Educación Digital, n¹ (2001), http://www.utpl. edu.ec/ried/images/pdfs/vol4-1/estrategias_desarrollo.pdf.

«Hoy inicia sus cursos la Universidad a Distancia». La Nación, 1 de julio de 1978.

«La situación financiera de la UNED». La Nación, 6 de agosto de 1977.

«La UNED: un nuevo reto académico». Acontecer Universitario, mayo-junio de 1992, nº18.

«La Universidad Estatal a Distancia». Libertad, 10-16 de junio de 1976.

León Villalobos, Edwin. Cuadernos del Pensamiento Universitario. Discursos. Heredia: Departamento de Publicaciones de la Universidad Nacional, 1985.

«Mejoramiento educativo atiende a grupos en estado de indigencia». Acontecer, marzo-mayo de 2008. 
Mora Vicarioli, Francisco. «Objetos de aprendizaje: importancia de su uso en la educación virtual». Revista Calidad en la Educación Superior, $\mathrm{n}^{\circ} 3$ (1), 104-118.

Moreno Castalleda, Manuel. Educación a distancia, un caleidoscopio para el aprendizaje en la diversidad, en Veinte visiones de la educación a distancia. Guadalajara: UDGVIRTUAL, 2012.

Oduber Quirós, Daniel. «La universidad llegará a todos los jóvenes del país». La Nación, 10 de marzo de 1977.

Pacheco Fernández, Francisco. «Grave crisis financiera obligará a cierre de la UNED». La Nación, 30 de julio de 1977.

Parreaguirre Camacho, Juan. Los centros universitarios en el sistema de educación a distancia, en Congreso internacional: tecnología y educación a distancia, El estudiante de la educación a distancia en la perspectiva de nuevo milenio X, Tomo II. San José: EUNED, 1999.

República de Costa Rica. Colección de leyes y decretos. San José: Imprenta Nacional, 1977.

Rodríguez, Cristián. «El crimen de la autodidáctica». La Nación, 2 de julio de 1976.

Ruiz, María y Salazar, Claudia. Educación virtual: plataforma de aprendizaje y desarrollo regional, en Congreso internacional: tecnología y educación a distancia. El estudiante de la educación a distancia en la perspectiva de nuevo milenio $X$, Tomo II. San José, EUNED, 1999.

Torres Rodríguez, José. «Los treinta años del Programa de Estudios Generales en la UNED». Acontecer, mayojulio del 2008.

«UNED: educación para todos», Suplemento publicitario especial «Empresas Exitosas». La Nación, 25 de abril del 2008.

«UNED responde a necesidades de comunidades indígenas centroamericanas». Acontecer, mayo-julio de 2008.

«UNED: Universidad de trabajadores». Acontecer, noviembre de 2007-febrero de 2008.

UNED. Primer Congreso Universitario. Informes de resultados de los talleres. San José: EUNED, 1988.

UNED. Congreso Universitario por un Cambio con Participación Real: Memoria. San José: EUNED, 2000.

UNED. Creación y organización de la Universidad Estatal a Distancia. San José: CIDREB, 2005.

UNED. Lineamientos de Política Institucional 2007-2011. San José: EUNED, 2007.

Vargas Solís, Luis. «El papel democratizante de la UNED en el contexto de la crisis social y económica», en Universidad Estatal a Distancia. Segundo Congreso Universitario. Por un cambio con participación real. San José: EUNED, 2000. 